\title{
Stability and lifetime study of carbon nanotubes as cold electron field emitters for electron cooling in the CERN extra low energy antiproton ring
}

\author{
Bruno Galante(1) \\ European Organization for Nuclear Research (CERN), Geneva 23, 1211, Switzerland; \\ The University of Liverpool, Liverpool, L69 3BX, United Kingdom \\ and The Cockroft Institute, Sci-Tech Daresbury, Warrington, WA4 4AD, United Kingdom \\ Gerard Alain Tranquille and Marcel Himmerlich $\odot$ \\ European Organization for Nuclear Research (CERN), Geneva 23, 1211, Switzerland \\ Carsten Peter Welsch $\odot$ \\ The University of Liverpool, Liverpool, L69 3BX, United Kingdom \\ and The Cockroft Institute, Sci-Tech Daresbury, Warrington, WA4 4AD, United Kingdom \\ Javier Resta López® \\ ICMUV-Institute of Materials Science, University of Valencia, 46980, Spain \\ and The University of Liverpool, Liverpool, L69 3BX, United Kingdom \\ and The Cockroft Institute, Sci-Tech Daresbury, Warrington, WA4 4AD, United Kingdom
}

(Received 29 July 2021; accepted 25 October 2021; published 9 November 2021)

\begin{abstract}
Electron cooling is a fundamental process to guarantee the beam quality in low energy antimatter facilities. In extra low energy antiproton, the electron cooler reduces the emittance blowup of the antiproton beam and thus delivers a focused and bright beam to the experiments at the unprecedentedly low kinetic energy of $100 \mathrm{keV}$. In order to achieve a cold beam at this low energy, the electron gun of the cooler must emit a monoenergetic and relatively intense electron beam. An optimization of the extra low energy antiproton electron cooler gun involving a cold cathode is studied, with the aim of investigating the feasibility of using carbon nanotubes (CNTs) as cold electron field emitters. CNTs are considered among the most promising field emitting material. However, stability data for emission operation over hundreds of hours, as well as lifetime and conditioning process studies to ensure optimal performance, are still incomplete or missing, especially if the purpose is to use them in operation in a machine such as extra low energy antiproton. This manuscript reports experiments that characterize these properties and ascertain whether CNTs are reliable enough to be used as cold electron field emitters for many hundreds of hours.
\end{abstract}

DOI: 10.1103/PhysRevAccelBeams.24.113401

\section{INTRODUCTION}

The extra low energy antiproton (ELENA) ring is the newest and most compact synchrotron decelerator at CERN with a circumference of only $30.4 \mathrm{~m}$ [1-3]. The main goal of ELENA is to further decelerate antiproton beams coming from the antiproton decelerator [4] from 5.3 MeV to kinetic energies as low as $100 \mathrm{keV}$. At these unprecedentedly low energies, the number of antiprotons trapped by the experiments is enhanced by up to two orders of magnitude [1].

\footnotetext{
*bruno.galante@cern.ch

Published by the American Physical Society under the terms of the Creative Commons Attribution 4.0 International license. Further distribution of this work must maintain attribution to the author(s) and the published article's title, journal citation, and DOI.
}

During the deceleration process the beam emittance, or transverse energy, increases due to adiabatic blowup, intrabeam scattering, and scattering with residual gas species in the beam pipe. This leads to losses and a poor-quality beam which is not very useful for the experiments. To counteract these effects, electron cooling [5] is applied to reduce the longitudinal and transverse energy spreads of the antiproton beam, leading to an increased phase-space density. This is achieved by reaching a thermal equilibrium between the hot ion beam and a cold medium, the electron beam, which is continuously renewed. In order for the cooling to be effective, the electron beam must fulfill specific features regarding beam current and beam energy [6-8]. The cooling process takes place twice during ELENAs beam cycle $[9,10]$ : (i) First cooling plateau, where the antiproton beam with kinetic energy $650 \mathrm{keV}$ (momentum $35 \mathrm{MeV} / \mathrm{c}$ ) is cooled by an electron beam 
with a kinetic energy of $355 \mathrm{eV}$ and a current of $5 \mathrm{~mA}$ and (ii) Second cooling plateau or low-energy extraction plateau, where the antiproton beam with kinetic energy $100 \mathrm{keV}$ (momentum 13.7 MeV/c) is cooled by an electron beam with a kinetic energy of $55 \mathrm{eV}$ and a current of $1 \mathrm{~mA}$.

In both cases, the electron beam has a diameter of $25 \mathrm{~mm}$ in order to fully enclose the antiproton beam of ELENA.

For better clarity, the typical ELENA cycle is illustrated in Fig. 1. As it is possible to see from the ELENA cycle, the electron cooler must work in switching mode to comply with the timing of the electron cooling pleateaus. In detail, the electron gun must emit for approximately $15 \mathrm{~s}$ during the first cooling plateau and for approximately $8 \mathrm{~s}$ during the second cooling plateau. During injection, deceleration steps and ejection the gun is switched off. Furthermore, the gun must emit for several hundreds of hours with an emission stability of at least $5 \%$, meaning that suitable lifetime and emission stability are critical. In this context, when describing current stability, we refer to current fluctuations in the mid- and long-term as small and local current outbursts do not represent an issue in the cooling process.

A thermionic electron source is normally operated in ELENA, although its performance is limited due to the relatively high-transverse energy of the emitted beam $(>100 \mathrm{meV})$ and the need to heat it to high temperatures makes its design and operation quite complicated. Photoemission has also been considered, but the low lifetime of the cathodes is incompatible with operation specifications. For such reasons, we are investigating the feasibility of using carbon nanotubes (CNTs) as electron field emitters, which would lead to a cold cathode electron gun. Despite field emission being quite a well-known effect since 1928 when first proposed by Fowler and Nordheim [11], it has recently become a fruitful field of research, thanks to the technological progress that has opened up the possibility of creating 2D nano-structures. 2D nano-materials can have greatly enhanced field emission

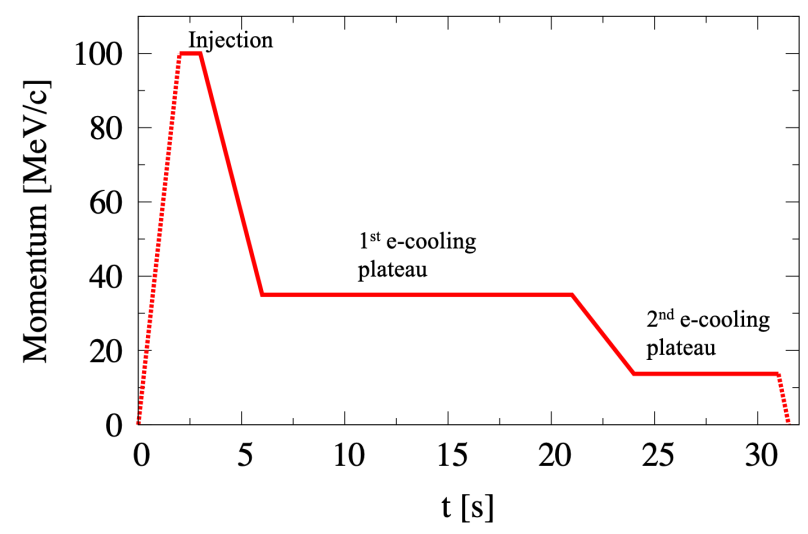

FIG. 1. Example of a typical beam cycle of the ELENA ring, showing the time evolution of the antiproton beam momentum. properties and permit to extract relatively high-current densities (usually dozens of $\mathrm{mA} / \mathrm{cm}^{2}$ ) at a relatively low-applied electric field (some $\mathrm{V} / \mu \mathrm{m}$ ). This is possible thanks to a field enhancement effect at the nano-structures' tips which experience a higher local electric field than the applied electric field. This effect permits a lower applied electric field to still emit large currents. The field enhancement is usually described by means of the so-called field enhancement factor $\beta$ [12].

For our study, CNTs have been chosen due to their remarkable properties in this field. They are nowadays considered among the most promising materials for field emission, capable of reaching high-current densities, being largely chemically inert and having a good emission stability. Several groups have reported promising results using arrays of vertically aligned CNTs. Such arrangement minimizes the screening effect which usually severely affects the performance of forests and disordered structures [13-17]. However, most of the existing literature on this topic is oriented to the study of the maximum reachable current, and there is very little known about emission stability and lifetime. According to several different studies, CNTs are able to reach extremely high-emitted current densities, even up to $100 \mathrm{~mA} / \mathrm{cm}^{2}$. However, such results are usually relative to quite small samples and, therefore, translate to relatively small nominal currents, in the range of $\mu \mathrm{A}$. Stability measurements are usually performed over short times (up to $24 \mathrm{~h}$ ) and comprehensive lifetime studies are seldom reported [18-21]. In our case, we aim at nominal currents in the range of some $\mathrm{mA}$, in order to prove that CNT samples can withstand the required ELENA electron beam currents and at the same time stably emit for hundreds or even thousands of hours.

In the next sections, we will go into the details of our research. In Sec. II, we describe the experiments performed and the experimental techniques used. In Sec. III, the focus moves to the achieved results and their discussion. In detail, we describe (i) The choice of two possible CNT array types selected for testing, (ii) The stability and lifetime measurements performed on the two different array types and the conditioning process required to achieve repeatable and optimal performance, (iii) The choice of the best array type in light of the experiments' results, (iv) The tests performed in switching mode to comply with the ELENA cycle, (v) The performance of a new chip with the selected array type operated under best conditions and a comparison study with the previous results, (vi) An emission stability study at different emitted current values to assess compliance with the gun requirements (current fluctuation of less than 5\%), and (vii) The surface analysis of the samples to evaluate the benefits of the conditioning process and further characterize the samples. Finally, in the conclusion, we focus on the concluding remarks while summarizing the research highlights. 


\section{EXPERIMENTS}

The main experimental setup shown in Fig. 2 is operated in diode configuration. It consists of a CNT array acting as the cathode. The emitted electron beam is collected and measured at a molybdenum plate which acts as the anode. The setup is placed in a vacuum chamber with three flanges dedicated to sample hosting.

Each flange can host three samples, giving a total of up to nine samples. Each sample is independent of the others and can have a diameter of up to $45 \mathrm{~mm}$. The flanges have undergone a few updates over the last two years. In the current version, the distance between cathode and anode is set to approximately $800 \mu \mathrm{m}$ and a mica insulating plate is placed on the top of the cathode to avoid contributions from the cathode edges. The mica plate acts as a mask as well, having a fixed aperture which permits precise control of the emitting area, Fig. 2(b).

Each sample stack is made of a copper support that acts simply as a holder, a Vespel or mica insulating plate, a stainless steel (SS) plate on top of which there is an $n$-doped Si substrate with the CNT array, and finally the mica insulating mask. The purpose of the SS plate is to make the electrical connection. This stack is kept together by means of PEEK insulating screws. Finally, a molybdenum plate is fixed directly on the copper support using ceramic washers and screws. The electrical connections are made by means of copper/Kapton wires; one connected to the SS plate and the second to the molybdenum plate, Fig. 2. Both wires are then connected to SHV feedthroughs. To produce the electric field, we apply a negative voltage on the cathode using an ISEG HPn-120256 with a maximum voltage of $-12 \mathrm{kV}$. The current measurements are made at the anode side, which is grounded, using a Hameg programmable multimeter HM8012 with resolution of $1 \mu \mathrm{A}$. Our samples are all relatively big in size, meaning that we are able to easily extract currents from hundreds of $\mu \mathrm{A}$ up to several $\mathrm{mA}$.

For the data acquisition we use LabView [22] routines, both for the DC and the switching mode. The pressure is monitored with a Pfeiffer full-range vacuum gauge able to measure from $1 \times 10^{-9}-1000$ mbar. Two types of CNT arrays have been characterized in this study: a honeycomblike array (CNT1) and a square-islands array (CNT2). Both geometries were characterized and validated with scanning electron microscopy (SEM) before operation. The scanning electron microscope used is a Zeiss XB540 FIB-SEM. Figure 3 shows the scanning electron micrographs of the two arrays. In both cases, the CNT height is of about $50 \mu \mathrm{m}$. The honeycomb-like structure pattern is made of an array of hexagons with side size of $10 \mu \mathrm{m}$ and spacing between hexagons of $5 \mu \mathrm{m}$. The CNTs, also visible in Fig. 3, are placed inside the hexagons, while the channels

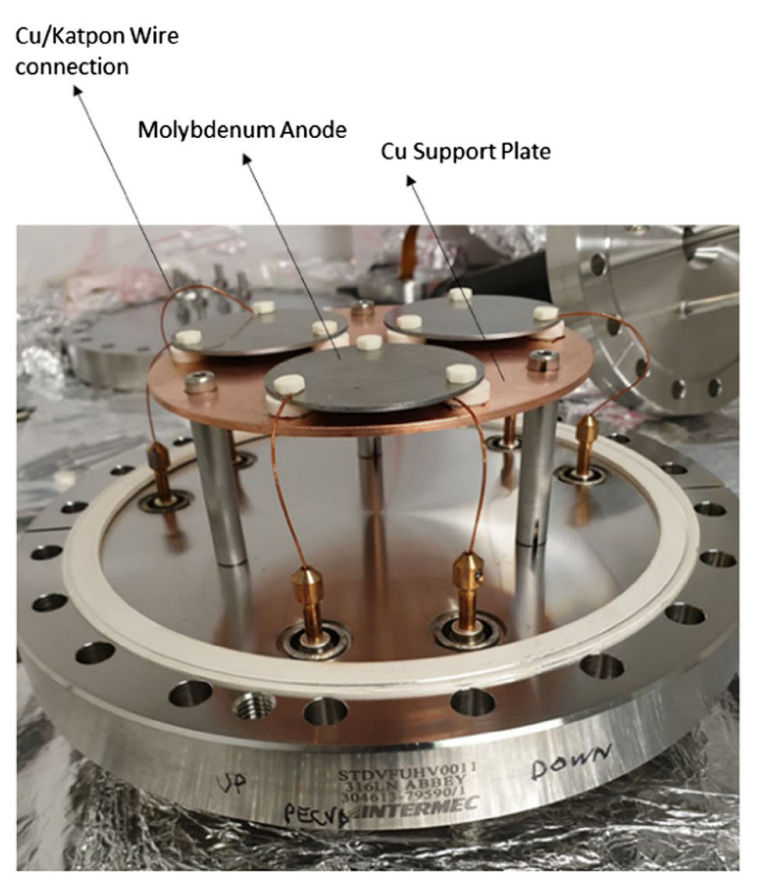

(a)

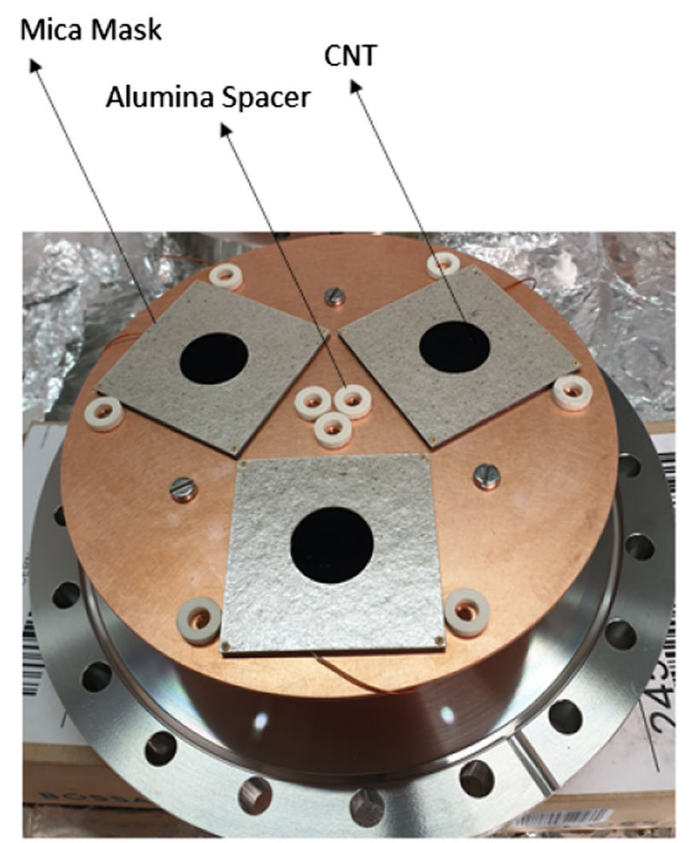

(b)

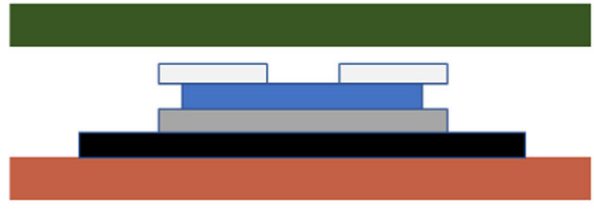

Mo Plate Mica Mask CNT SS Plate Vespel Plate Cu Support

(c)

FIG. 2. (a) Cold cathode test bench. (b) Flange and CNT samples arrangement. (c) Sample stack layout. 


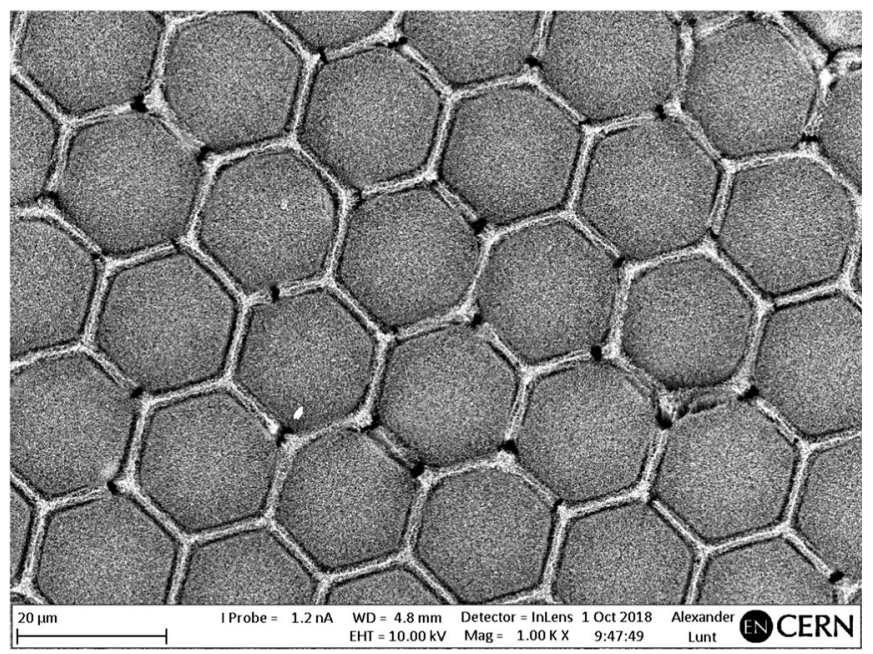

(a)

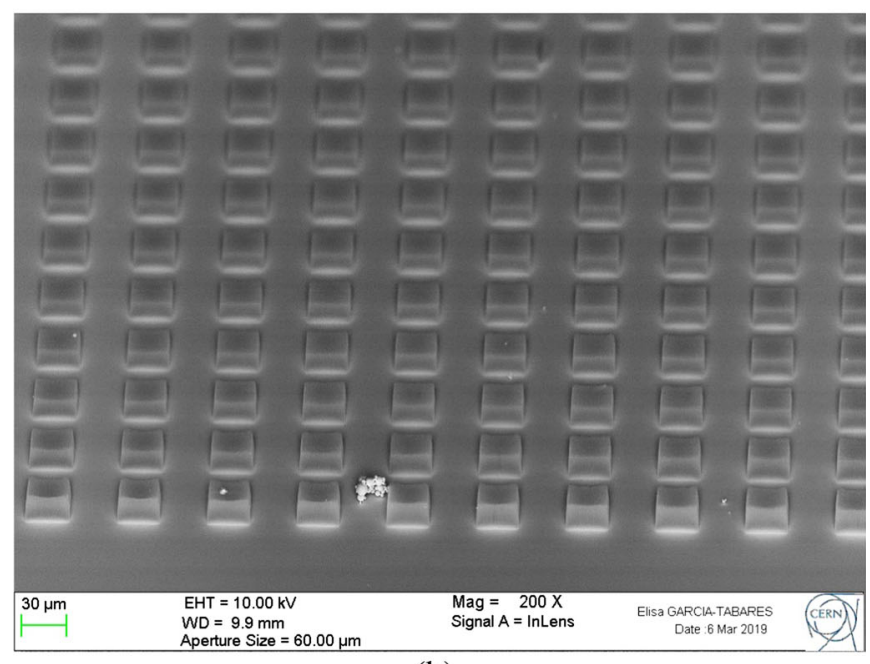

(b)

FIG. 3. SEM images of the honeycomb-like array CNT1 (a) and squared-islands array CNT2 (b).

separating the hexagons are empty. The square-islands array pattern is made of $30 \times 30 \mu \mathrm{m}$ squares, with a spacing of $30 \mu \mathrm{m}$. In this case, the CNTs constitute the squares while the channels between the islands are empty. The samples have been purchased from an external commercial supplier, Nanolab Inc. [23], and they are $8 \mathrm{~mm}$ in diameter. All samples were grown using thermal chemical vapour deposition [24] using a combination of Al and $\mathrm{Fe}$ as catalysts to promote CNT growth. The patterns have been selected to limit screening effects, thus increasing the emitted current density, while still having a large number of emitters participating in the emission. This minimizes the emitted current per single tip at the nominal required current value. Several tests have been conducted to characterize various key properties: emitted current as a function of the applied electric field, emission stability, lifetime, switching, and measurements of beam energy.
A commercial ultrahigh vacuum (UHV) system, with a base pressure below $2 \times 10^{-10}$ mbar (SPECS Surface Nano Analysis $\mathrm{GmbH}$, Berlin, Germany), is utilized for secondary electron yield (SEY), x-ray photoelectron spectroscopy (XPS), and ultraviolet photoelectron spectroscopy (UPS) characterization of CNT samples on silicon. The system consists of a hemispherical electron energy analyzer with nine channeltrons (Phoibos 150), a monochromated $\mathrm{AlK} \alpha \mathrm{X}$-ray source (XR50 M with Focus 500, $h \nu=$ $1486.7 \mathrm{eV}$ ), and a noble gas discharge source that was operated with He (UVS 10/35, $h \nu=21.2 \mathrm{eV}$ ). The energy scale of the analyzer is regularly calibrated using sputtercleaned polycrystalline $\mathrm{Cu}, \mathrm{Ag}$, and $\mathrm{Au}$ foils. For analysis of the SEY between 0 and $1800 \mathrm{eV}$ primary electron energy, an electron beam of $\sim 2 \mathrm{nA}$ generated by a Kimball Physics ELG-2 electron gun at a distance of $2 \mathrm{~cm}$ to the sample was focused to a spot diameter of $1 \mathrm{~mm}$ on the surface. The details of the setup and the implemented experimental conditions are described in Ref. [25], a sample bias of $\pm 47.1 \mathrm{~V}$ was used. The samples were characterized as loaded from ambient temperature as well as after several heating cycles at constant temperature in the UHV chamber $\left(180{ }^{\circ} \mathrm{C}\right.$ for $30 \mathrm{~min}, 180{ }^{\circ} \mathrm{C}$ for $120 \mathrm{~min}$, $250{ }^{\circ} \mathrm{C}$ for $240 \mathrm{~min}$, and $300^{\circ} \mathrm{C}$ for $180 \mathrm{~min}$ ) with $30 \mathrm{~min}$ of ramp-up and ramp-down time, utilizing a radiative heater placed on the backside of the sample holder, and subsequent cooldown. The maximum pressure rise during heating was by two orders of magnitude. For detailed analysis of the degassing species during the annealing cycles, the residual gas composition of the vacuum chamber was monitored using a Pfeiffer PrismaPro quadrupole mass spectrometer. Besides the structured samples, two plane $\mathrm{Si}$ wafers have been overgrown with CNTs using the comparable processing conditions to allow large-scale analysis of homogeneous CNT samples by the surface spectroscopy methods, which have a spot diameter of $1-2 \mathrm{~mm}$.

\section{RESULTS AND DISCUSSION}

\section{A. Lifetime and stability measurements}

The characterization of several CNT1 and CNT2 type samples started with a conditioning and stability test at fixed field before the voltage was slowly ramped up. These initial procedures as well as the results from vacuum monitoring showed that the emitted current is strongly dependent on the vacuum level and vice versa, Fig. 4. This can be partly explained by the shortening of CNTs during current peaks and outgassing. Other important factors are ion bombardment and residual gas ionization, effects that can become dominant if the pressure is not low enough. Current emission can be significantly affected by ion bombardment and ionization processes leading to breakdowns, thus affecting the stability of the emission and in some cases altering the field emitter tips. This also disrupts 


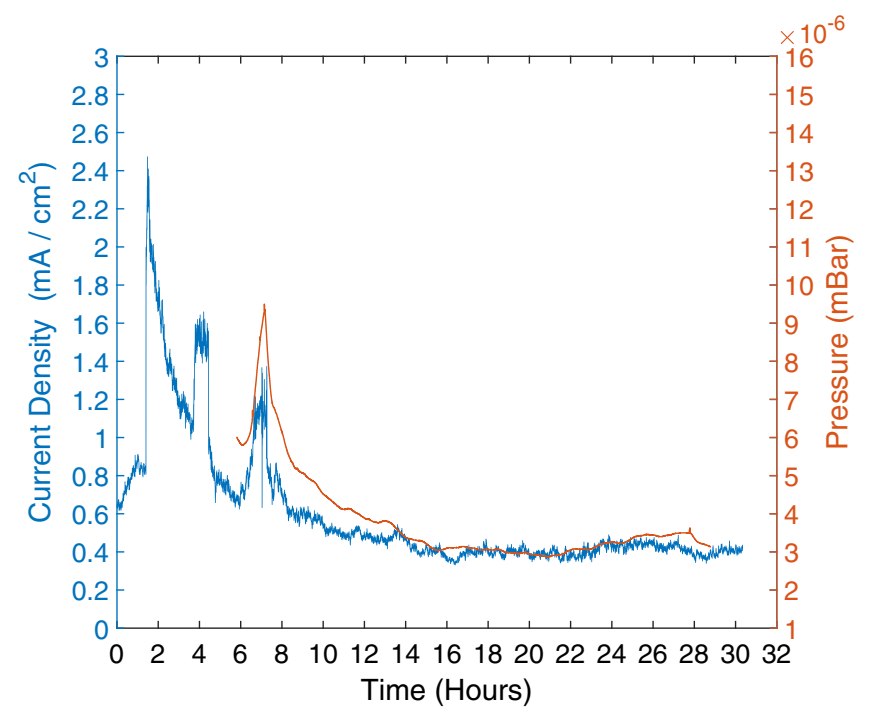

(a)

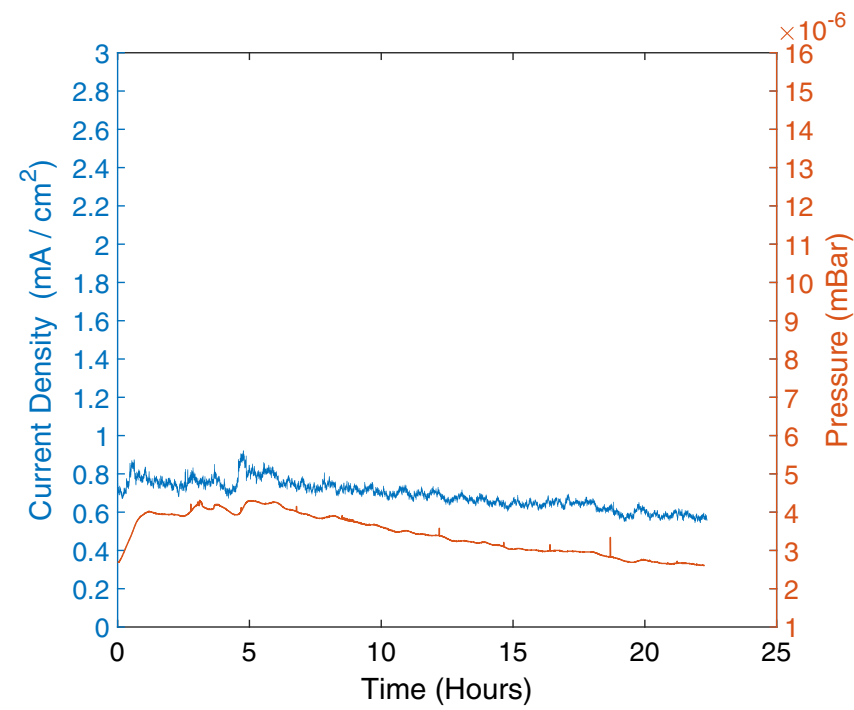

(b)

FIG. 4. Conditioning test for CNT1-type array. Current density and pressure as a function of time for field $E=1.94 \mathrm{~V} / \mu \mathrm{m}$ (a) and $E=2.4 \mathrm{~V} / \mu \mathrm{m}$ (b).

the field emission properties of the material and leads to degradation [26]. These last two effects play a major role in affecting emission stability and lifetime. Most of these phenomena act in a cause and effect dynamics. This means that in some cases the deterioration of the CNTs depends on the pressure, due to ion bombardment and outgassing inducing shortening of the CNTs and arcing. In other cases, the pressure depends instead on current spikes, arcing, and outgassing too. Hence, in order to limit these cause and effect dynamics and ensure a stable and reliable emission a good vacuum and a slow conditioning process are essential. For instance, a slow conditioning process with gradual increase of the electric field (and consequently of the emitted current) is also beneficial to shorten, and/or burn, protruding CNTs that can alter the overall cathode performance affecting the emission stability. SEM imaging was conducted before the tests and after a few hundreds of hours of operation, but did not show any clear sign of deterioration, indicating that if a shortening of the CNTs did happen it was negligible or marginal. The major changes to the emission performances can then be attributed to burnout/shortening of protruding CNTs, which is not trivial to detect via SEM imaging, outgassing, and desorption from the nanotubes' tips.

The emission properties as a function of the applied electric field have also been studied, Fig. 5. The threshold field, defined here as the electric field necessary to reach a current density of $1 \mathrm{~mA} / \mathrm{cm}^{2}$, is $E_{\text {tr }}=1.67 \mathrm{~V} / \mu \mathrm{m}$ for the two cases studied. The two cases mentioned correspond to two different voltage ramps performed on a CNT1 sample: the first one was made after about $380 \mathrm{~h}$ of operation and the second one after an additional $300 \mathrm{~h}$. However, the behavior of the two curves, both fitted with the classic Fowler-Nordheim (FN) equation [11] for simplicity, shows a slight shift when the electric field increases, indicating that the conditioning process has indeed changed the emission properties (Fig. 5). The classic FN equation used is the following:

$$
J=\left(A E_{L}^{2} / \Phi\right) \exp \left(\frac{-B \phi^{3 / 2}}{E_{L}}\right)
$$

where $J$ is the current density, $A=1.54 \times 10^{-6} \mathrm{AeVV}^{-2}$, $B=6.83 \times 10^{9} \mathrm{eV}^{-3 / 2} \mathrm{Vm}^{-1}, \Phi$ is the work function of

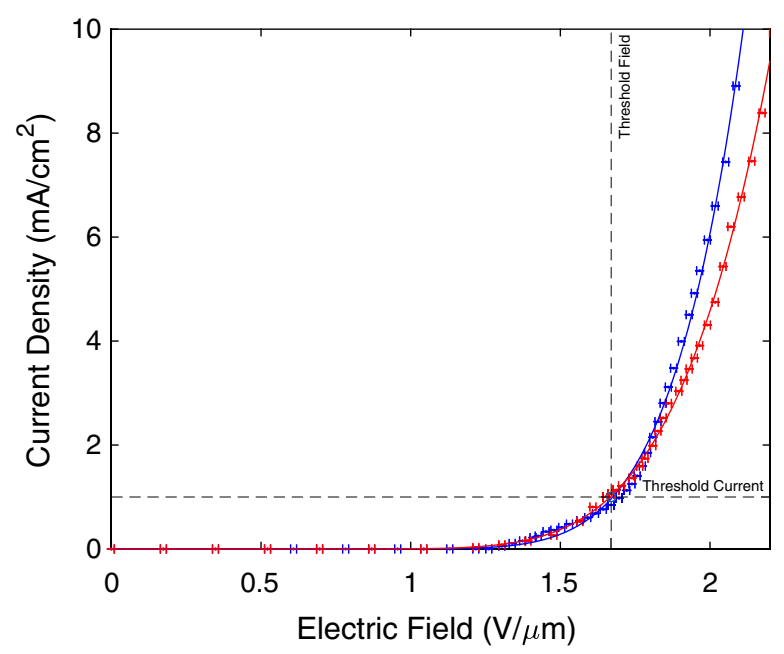

FIG. 5. Plot of experimental points and fit with FowlerNordheim equation of an electric field ramping test for CNT1type array. On the axis: applied electric field vs current density at $t_{1}=380 \mathrm{~h}$ (blue) and $t_{2}=t_{1}+300 \mathrm{~h}$ (red). The threshold field relative to the threshold current of $1 \mathrm{~mA}$ is represented with the dotted lines and centered at the value $E_{\mathrm{tr}}=1.67 \mathrm{~V} / \mu \mathrm{m}$. The error bars for Plots 1 and 2 are derived from the instruments' accuracy, e.g., the multimeter sensitivity for the current density and the voltage power supply sensitivity for the electric field. 
the material, and $E_{L}$ is the local field at the emitting tip. $E_{L}=\beta E$, with $E$ being the applied electric field and $\beta$ the field enhancement factor [12,27]. Despite the FN formula being theoretically incorrect when it comes to describing the field emission properties of CNTs [28-30], it is still useful for performance and threshold field comparison due to its fitting simplicity.

Further tests have shown that the CNT1 samples can emit for hundreds of hours without showing signs of burnout.

Concerning the test with CNT2-type arrays, most chips did not show acceptable performances. Among the best features shown by the CNT2s, we can point out a higher threshold field, $E_{\mathrm{tr}}=2.71 \mathrm{~V} / \mu \mathrm{m}$, with respect to the CNT1s. On the other hand, although CNT2s showed a good initial stability, their lifetime was significantly lower than that for CNT1s, as shown in Fig. 6. In their best performance, the CNT2s reached total burnout after approximately 500 operation hours, a value much smaller than for the CNT1s, for which an operational time of more than $1500 \mathrm{~h}$ has been demonstrated without clear signs of burnout.

The $1500 \mathrm{~h}$ value concerning the CNT1-type samples' operation time arises from a cumulative analysis of all the measurements performed on the CNT1 sample during the past two years. These tests include trial voltage ramps and several conditioning tests made at fixed fields and performed at different base pressures. Such additional tests are similar to the tests presented so far and are not directly reported here since they do not bring any additional value nor further depth to the discussion.

In the CNT2-type arrays, the number of CNTs is significantly smaller than it would be in a CNT1-type array of equal size. For equal chip size, a CNT2-type array would have a number of CNTs of around 25\% compared to a CNT1-type array. This explains why the field necessary to extract a comparable amount of current is necessarily higher. As a consequence, this causes two operational problems: a bigger current per tip must be emitted, therefore increasing the stress on the nanotubes, thus negatively affecting stability and lifetime, as indicated by the experiments conducted so far; a larger applied electric field is required, which translates into more energetic electrons, while for our purpose it is important to keep the electron beam energy as low as possible.

All the above conceptual and experimental evidences convinced us to discard the CNT2-type arrays and focus our investigation on the CNT1-type arrays to be applied in the electron cooler gun.

The effect of the environment conditions on the current emission is also shown in Fig. 6. At the time of CNT2 burnout, a big vacuum spike can be noted as shown in Fig. 6(b). This is the same effect seen in the lifetime measurement of CNT1, Fig. 6(a). The two chips were being tested simultaneously and after the vacuum spike, the

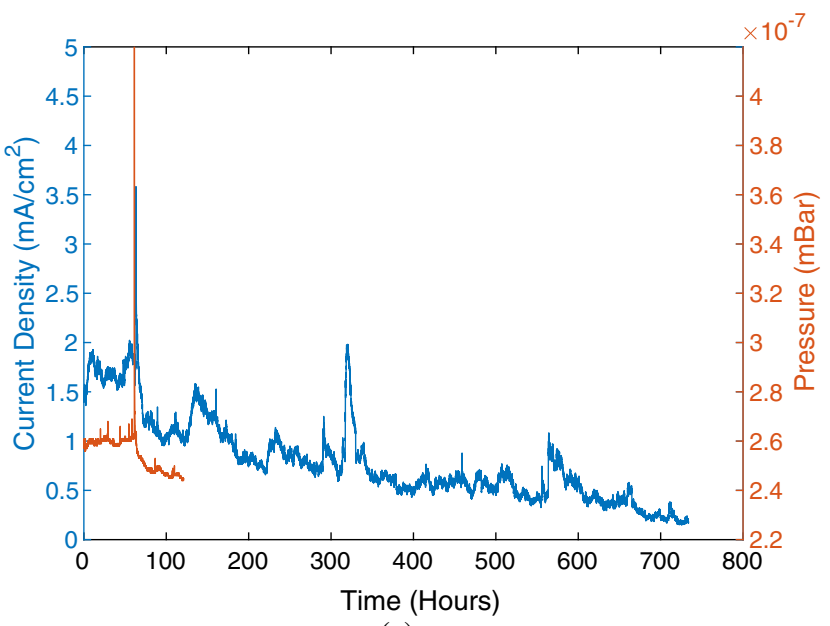

(a)

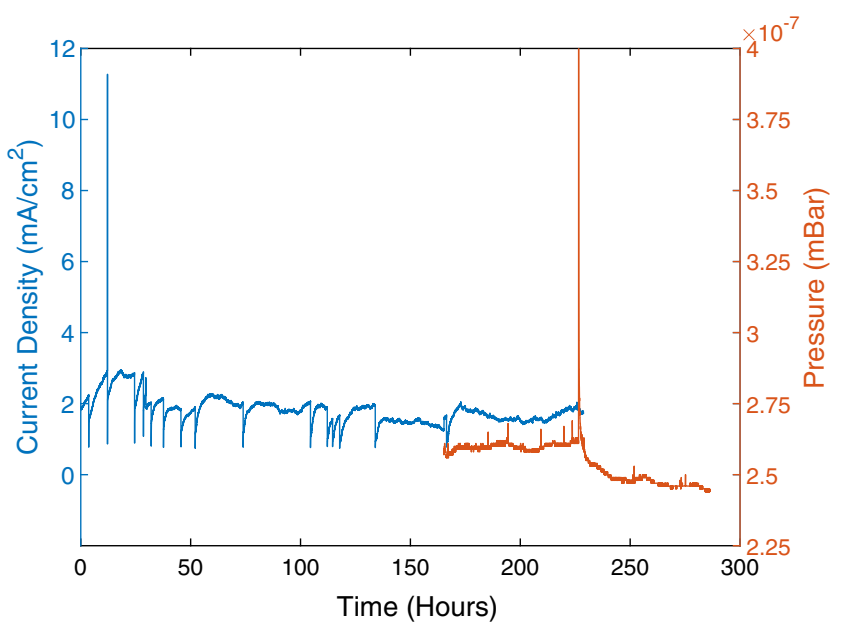

(b)

FIG. 6. Lifetime test for CNT1 with a fixed field $E=3 \mathrm{~V} / \mu \mathrm{m}$ (a) and for CNT2 with fixed field $E=3.9 \mathrm{~V} / \mu \mathrm{m}$ (b).

emitted current for the first array undergoes a steep peak and then drops significantly.

Further studies at different vacuum levels on other samples showed how the difference in emission stability can be striking. For optimal performance, a pressure in the order of $10^{-8}$ mbar or lower is necessary to avoid significant contributions from ionization processes. Further proof of this behavior is given by the increased performance of CNT1s when the pressure was decreased below $1 \times 10^{-8}$ mbar. It is indeed possible to infer from Figs. 7 and 8 how the emission stability was improved without clear signs of deterioration. As an additional improvement, a bakeout process was also added to clean the vacuum chamber and the emission region. Figure 7 shows results from stability tests conducted on the same CNT1 sample on which the lifetime and stability studies were previously reported. In this case, the test is performed in switching mode to test the feasibility of current switching as required for ELENA. In this mode, the cathode emits for about $15 \mathrm{~s}$, 


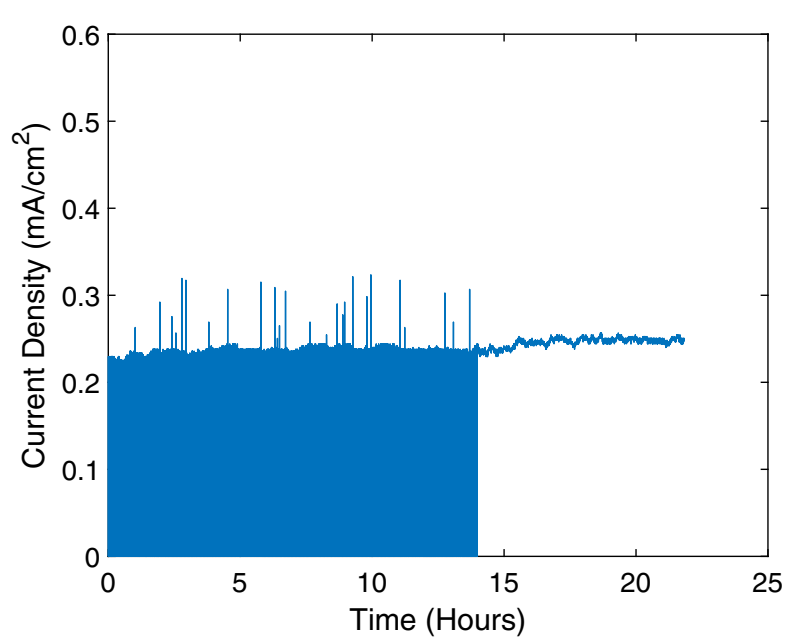

(a)

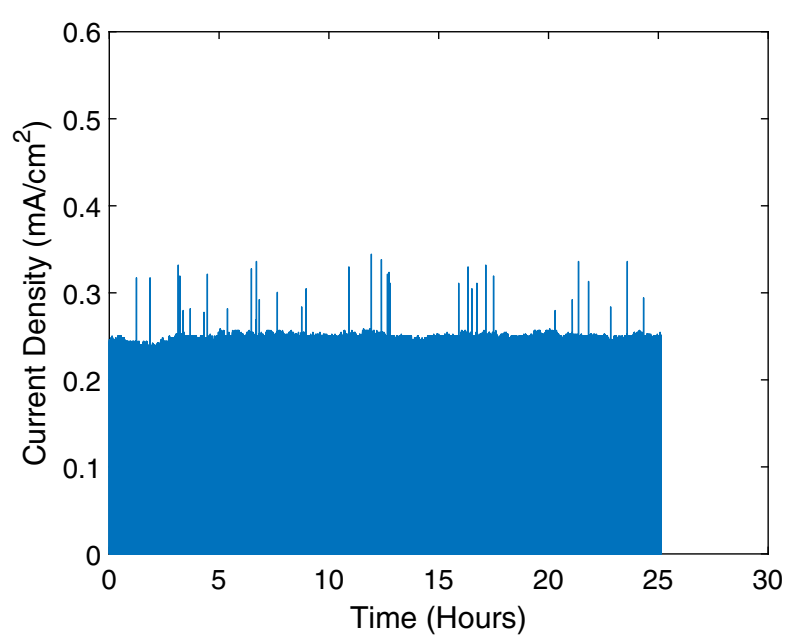

(b)

FIG. 7. Emission stability test in switching mode for a CNT1type array $(E=2 \mathrm{~V} / \mu \mathrm{m})$. In (a), the switching mode is operated for around $14 \mathrm{~h}$, then the emitter is subsequently operated in dc mode to compare stability. In (b), the switching mode is continued for an additional $25 \mathrm{~h}$.

which is approximately the cooling time for the first cooling plateau in ELENA, followed by $15 \mathrm{~s}$ of pause, which is approximately the time required for injection, deceleration steps, and ejection in the ELENA cycle. The emission switching was initiated via a LabView routine, which controls the power supply providing the negative voltage to the cathode. The power supply was switched on and off every $15 \mathrm{~s}$. In Fig. 7(a), it is possible to see how the switching does not cause significant variations despite the presence of local low current peaks. After around $14 \mathrm{~h}$, the switching was stopped to evaluate the stability in dc. Despite an initial slight increase in current, the stability was still optimal. In Fig. 7(b), the switching is restarted and continued for 25 additional hours without clear signs of degradation.

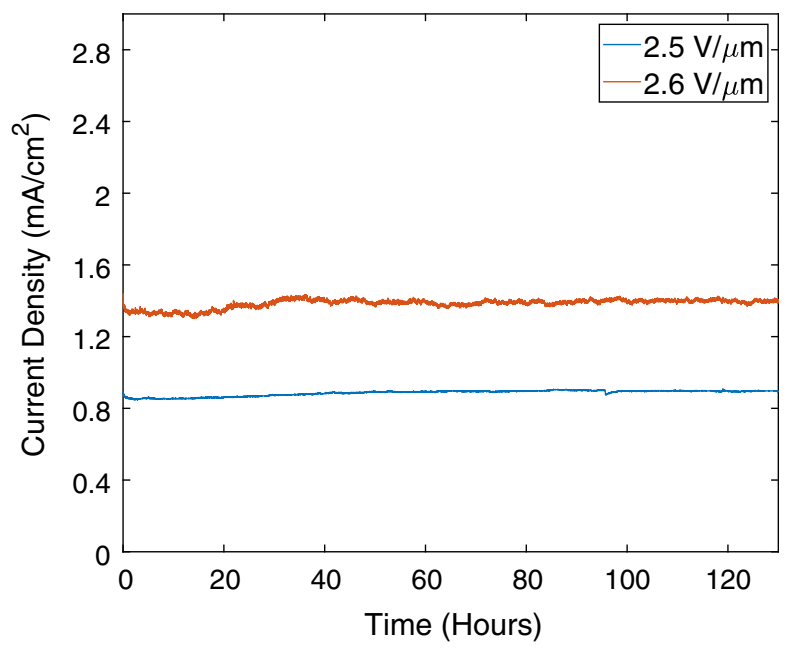

FIG. 8. Emission stability test for CNT1-type array at two different applied electric fields: $E=2.5 \mathrm{~V} / \mu \mathrm{m}$ and $E=2.6 \mathrm{~V} / \mu \mathrm{m}$.

In Fig. 8, the performance of another sample (CNT1type) is presented. The emission is remarkably stable for more than $250 \mathrm{~h}$ in total at two different applied electric fields, particularly at the lower field. In this case, there was an optimal pressure of less than $1 \times 10^{-9}$ mbar and a bakeout process at $220{ }^{\circ} \mathrm{C}$ was performed before the test. However, CNTs can easily be heated to much higher temperatures since they are grown at temperatures of the order of $700{ }^{\circ} \mathrm{C}$.

Comparing now the results obtained for the CNT1-type array, it is possible to extract some further evidence of the importance of the conditioning process and the need to ramp-up the electric field gradually. In fact, it could be seen in Fig. 5 that the current emitted per applied electric field is extremely high as compared to all other results from stability tests for the same sample. Especially considering that the results for the additional CNT1-type sample are comparable with the first sample's latest results. After efficient conditioning and with a proper base pressure of at least $10^{(-8)}$ mbar these discrepancies disappear. In fact, in Fig. 4(b) the extracted current density amounts to $0.7 \mathrm{~mA} / \mathrm{cm}^{2}$ on average for an electric field of $2.4 \mathrm{~V} / \mu \mathrm{m}$, and in Fig. 8 we have a similar current density of $0.9 \mathrm{~mA} / \mathrm{cm}^{2}$ at an electric field of $2.5 \mathrm{~V} / \mu \mathrm{m}$. This shows that samples of the same type roughly exhibit the same performance. These comparisons show that without a proper and efficient conditioning process it is hard to separate the real performance of the sample from spurious effects caused by ionization processes, adsorbates temporarily changing the work function value and presence of protruding CNTs, all factors that can significantly alter the emission properties.

We have conducted the same measurement of current density vs applied electric field on a second CNT1-type sample. In this case, the conditions were optimal for both pressure and bakeout. It is possible to see from Fig. 9 how, 


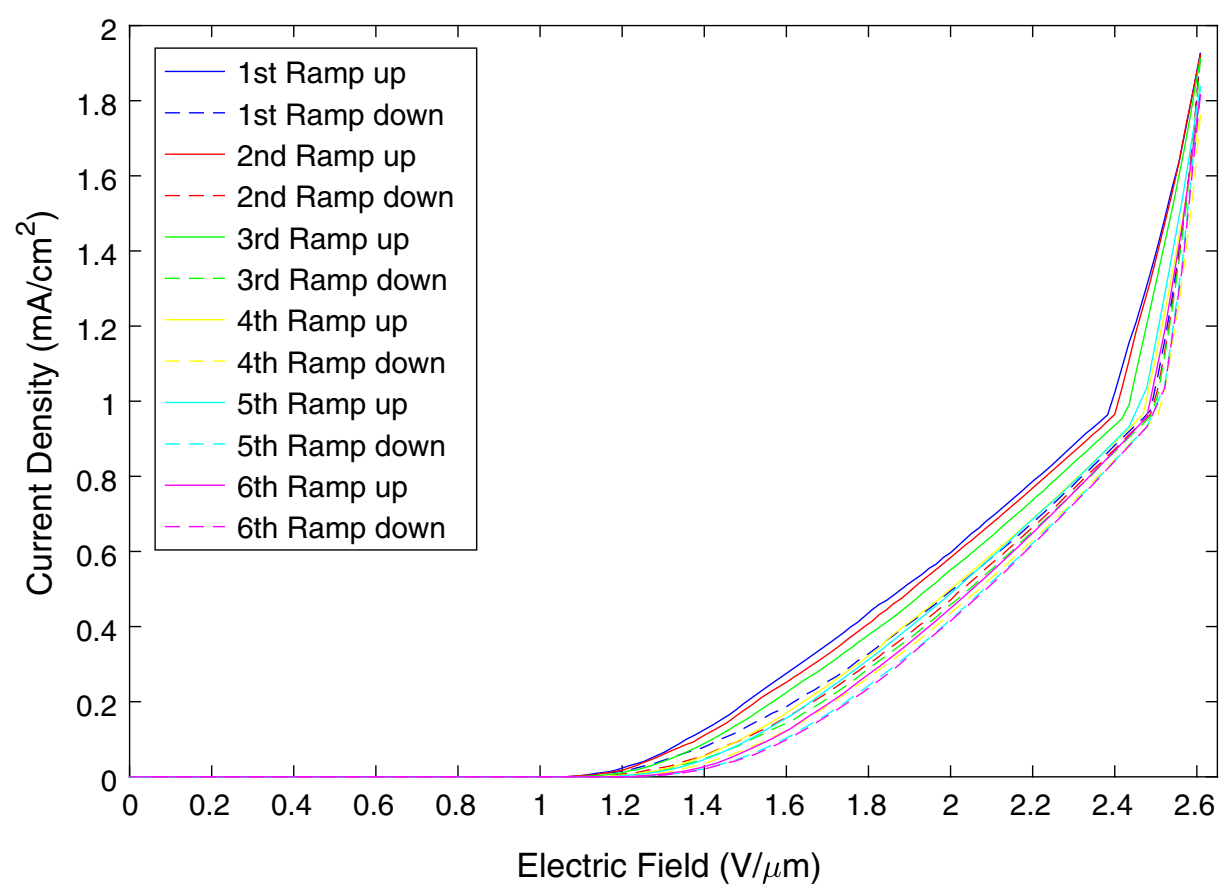

FIG. 9. Current density vs applied electric field for CNT1-type array. The test include six voltage ramps with data acquisition on both the way up and down. The electric field was ramped from 0 to $2.6 \mathrm{~V} / \mu \mathrm{m}$.

despite some small variations, the results tend to converge to similar values and also agree more with the results achieved via stability measurements. In this particular case, the electric field was ramped up and down 6 times from 0 to $2.6 \mathrm{~V} / \mu \mathrm{m}$. This electric field range allowed us to reach current densities up to $2 \mathrm{~mA} / \mathrm{cm}^{2}$. Since the emission target is indeed in the order of $1-2 \mathrm{~mA} / \mathrm{cm}^{2}$, we decided to limit the study to this range. This is also motivated by the same behavior shown in every ramp despite some little adjustment, which suggests that the conditioning process has been efficient and that the current values that were set as requirements are easily reachable at relatively low fields and in a reproducible way. In Fig. 9, it is also possible to notice a kink in the emitted current when the applied electric field exceeds $2.4 \mathrm{~V} / \mu \mathrm{m}$. This behaviour holds for all six ramps, both on the way up and on the way down, indicating the possible presence of a change in the emission behavior. This change seems to suggest the presence of two different emission regions, one below approximately $2.4 \mathrm{~V} / \mu \mathrm{m}$ where the current emission seems to be inhibited and one at higher fields where the exponential behavior of the current rise is enhanced.

In order to assess whether the stability is affected by the emitted current, we performed a further experiment on this last CNT1 sample. We studied the emission stability at five different applied electric fields for about 20 h, Fig. 10(a), and then calculated the standard deviation $\sigma$ for the emitted current density for each case. The standard deviation reflects the intensity of the current density fluctuations over the period of study. The results show an increase in the overall standard deviation when the current density increases. However, the calculation of the coefficient of variation percent shows instead an inverted trend. The coefficient of variation $c_{V}$, or relative standard deviation, is defined as the ratio between the standard deviation $\sigma$ and the mean value of the data set (i.e., the average current density $\langle J\rangle$ ) and it is usually represented as a percentage [31],

$$
c_{V}[\%]=\frac{\sigma}{\langle J\rangle} \times 100
$$

In this case, it represents the variation percentage of the emitted current density and provides a description of the emission stability over time. The analysis of the coefficient of variation shows how the current density variation percentage decreases at higher emitted current densities, indicating optimal stability when the emitted current density is increased, Fig. 10(b).

The experimental data is represented with circles, while the full line represents a fit of the experimental data in order to make the trend clearer. The fit was made with MATLAB and with a power equation of the type: $y=a x^{b}+c$.

This study suggests that CNTs can also stably emit at high-applied electric fields with coefficient of variation of even less than $1 \%$. These results are summarized in Table I.

Therefore, the choice of having large or small area cathodes would solely depend on the operational requirements regarding the total nominal current variations and most importantly the beam energy. For the emitted current and its variations, at equal nominal emitted current, a large area cathode permits to emit with lower current densities, 


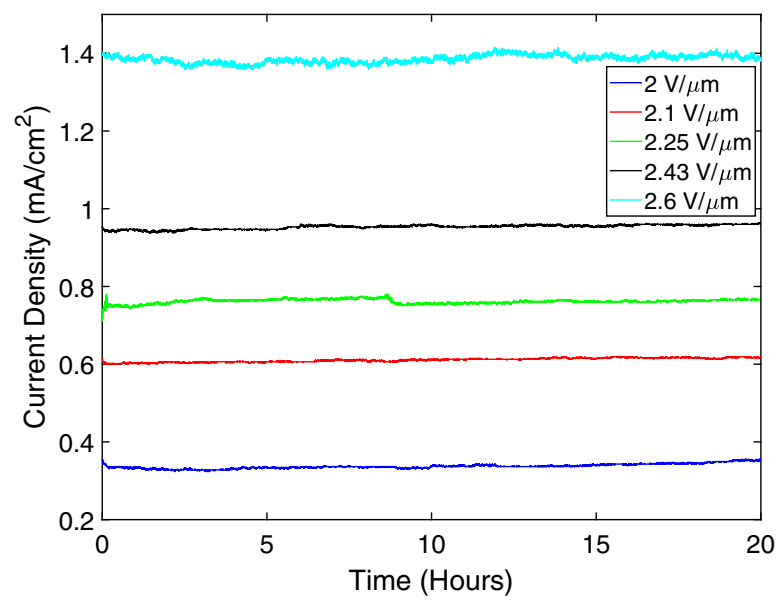

(a)

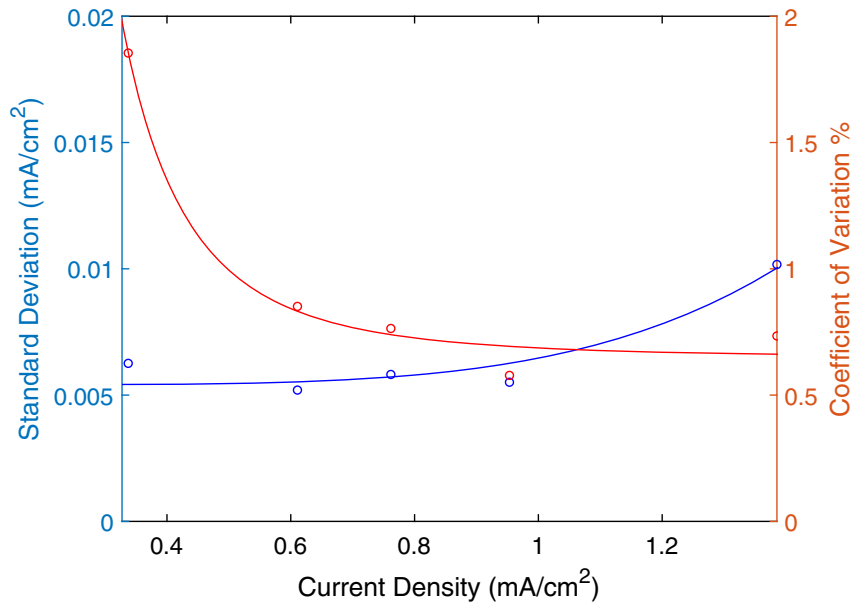

(b)

FIG. 10. (a) Current density $\left(\mathrm{mA} / \mathrm{cm}^{2}\right)$ as a function of time (h) for CNT1 sample. Measurement conducted at five different applied electric fields for $20 \mathrm{~h}$ of emission each. (b) Standard deviation $\left(\mathrm{mA} / \mathrm{cm}^{2}\right)$ and coefficient of variation $(\%)$ as a function of the current density $\left(\mathrm{mA} / \mathrm{cm}^{2}\right)$ for CNT1 sample at five different emitted current densities (five different applied electric fields).

therefore having slightly smaller nominal fluctuations; a behaviour that, however, does not extend to the current density variations percentage. For the beam energy, large area samples permit the application of a lower electric field at equal nominal emitted current, therefore having electrons with lower longitudinal kinetic energy. This could be beneficial for operations requiring low-energy electrons such as the ELENA experiment.

\section{B. Surface analysis and work function measurements}

Field emission strongly depends on the work function, it is therefore important to clearly determine its correct value. In fact, even localized changes in the work function due to the presence of adsorbates on the CNT tips can lead to
TABLE I. Measurement of standard deviation $\sigma$ and coefficient of variation $c_{V}$ of the emitted current density of a CNT1 sample at five different applied electric fields $E$ and current densities $\langle J\rangle$.

\begin{tabular}{lccc}
\hline \hline $\mathrm{E}(V / \mu \mathrm{m})$ & $\begin{array}{c}\text { Average } \\
\text { current density } \\
\langle J\rangle\left(\mathrm{mA} / \mathrm{cm}^{2}\right)\end{array}$ & $\begin{array}{c}\text { Standard } \\
\text { deviation } \sigma \\
\left(\mathrm{mA} / \mathrm{cm}^{2}\right)\end{array}$ & $\begin{array}{c}\text { Coefficient } \\
\text { of variation } \\
c_{V}(\%)\end{array}$ \\
\hline 2 & 0.337 & 0.006 & 1.85 \\
2.1 & 0.611 & 0.005 & 0.85 \\
2.25 & 0.762 & 0.006 & 0.76 \\
2.43 & 0.953 & 0.006 & 0.58 \\
2.6 & 1.385 & 0.01 & 0.73 \\
\hline \hline
\end{tabular}

drastic changes in the emitted current. Such changes will of course depend on the adsorbed elements since this can cause an increase in the work function. This translates into a decrease in the emitted current, or alternatively, an increase in the emitted current due to a drop in the work function $[32,33]$.

In order to assess these aspects, the surface properties of CNT samples were characterized under UHV to determine the surface composition by XPS and to characterize the global work function of the CNTs by SEY measurements and UPS. Structured samples with either the honeycomblike array or the square-islands array were characterized after loading in the analysis system. As the lateral resolution of the implemented experimental techniques is limited to a spot diameter of 1-2 $\mathrm{mm}$, leading to a superposition of signals from the CNT regions and the surrounding $\mathrm{Si}$ wafer, we have also characterized two CNT samples ("Full Coverage 1" and "Full Coverage 2"), which consisted of a Si substrate fully covered with CNTs on an area of $10 \times 10 \mathrm{~mm}^{2}$ that were grown using comparable processing parameters, except for slight adjustments in the gas ratio during growth. Additionally, on these two samples annealing cycles under UHV were performed to investigate whether air exposure affects the work function and surface composition and whether a thermal treatment in vacuum could improve the surface quality. All measurements were performed at room temperature, i.e., after each annealing step, the sample was left to cool down. Several groups have reported results on the work function of CNTs, with values spanning from 4 to even $5.6 \mathrm{eV}$, depending on the technique used and possible alterations due to adsorbates [34-36]. The performed measurements aim to quantify the work function value of multiwalled CNTs before and after annealing, which is linked to their practical utilization in a baked UHV chamber for field-emission applications in ELENA.

Residual gas analysis (RGA) was performed during each annealing cycle by means of a continuous recording of spectra to monitor the outgassing and desorption. This allows the simulation of a bakeout of the vacuum chamber to improve the base pressure, while simultaneously also heating the CNT emitter region. The performed RGA 
measurements inevitably includes signals from species desorbing from the CNT area as well as the predegassed sample holder. Therefore, their results allow only a partial identification of bakeout induced benefits for the CNT surface. As expected, mainly $\mathrm{H}, \mathrm{H}_{2}, \mathrm{O}, \mathrm{OH}, \mathrm{H}_{2} \mathrm{O}, \mathrm{CO}$, various hydrocarbons, and $\mathrm{CO}_{2}$ are desorbed. During the first cycle at $180{ }^{\circ} \mathrm{C}$, the main contribution is water desorption and emission of volatile species. At higher annealing temperatures the relative content of $\mathrm{CO}$ and $\mathrm{CO}_{2}$ increases, which is consistent with desorption and conversion of species at these temperatures. We anticipate that such a thermal treatment would be able to successfully clean the sample and especially the CNTs for electron emission. As a result, it would also prevent pressure bursts during emission ramp-up as confirmed by our stability measurements. The RGA experimental results hereby presented cannot provide for a quantitative analysis relative to the thermal treatment benefits, although they do provide for a qualitative endorsement of its beneficial effect to the emission region and sample cleaning.

XPS measurements of the sample "Full Coverage 2" before and after the annealing process are shown in Fig. 11. Most important to note is the low-impurity level of oxygen (1.1 at.\%) even without annealing, for which the weak signal of the $\mathrm{O} 1 \mathrm{~s}$ state is at $532.5 \mathrm{eV}$ binding energy (i.e., the binding energy of the electrons in the first orbital, the orbital "1s", of the oxygen atom). This oxygen content dropped further to below the detection limit of the $\mathrm{O} 1 \mathrm{~s}$ state $(0.1$ at. \%) after annealing. In addition, iron $(\mathrm{Fe})$ is detected $(0.05$ at.\%) which is due to the $\mathrm{Fe}$ catalyst layer that is deposited onto the $\mathrm{Si}$ wafer prior CNT growth. The fluorine impurity signal ( 0.4 at.\% before heating) disappeared as well as the oxygen contribution after the thermal processing. Figure 11(b) includes the spectra of the C1s state (i.e., the binding energy of the electrons in the first orbital, the orbital " $1 \mathrm{~s}$ ", of the carbon atom) for the sample prior to and

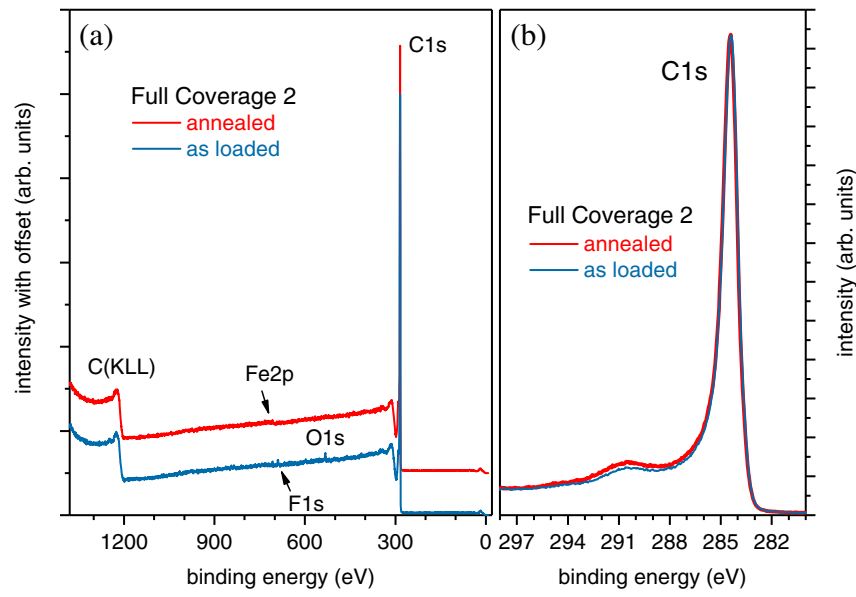

FIG. 11. (a) XPS survey spectra of CNT sample "Full Coverage 2" as loaded and after the complete UHV annealing process. (b) Corresponding $\mathrm{C} 1 \mathrm{~s}$ spectra. after the annealing steps. Overall, only slight changes in the XPS spectra occurred. While the peak maximum at $284.5 \mathrm{eV}$ is unchanged, the thermal processing in UHV induced a slight signal increase of the electronic excitations (around $290.5 \mathrm{eV}$ ) of the CNT sample. We attribute this aspect to the thermally induced desorption of adsorbates from the nanotube surfaces that partially saturated $\pi$ electrons before the heating process. It is anticipated that the increase of delocalized electrons at the CNT surface is advantageous for its electron emission performance and stability.

The work function was extracted from the low-primary energy part of the SEY curve. The method used, called beam-stop method, was already applied in earlier studies $[25,37,38]$ and is correct when the beam is impinging normally to the surface. As this method requires a reference material for determining the work function, we measured in parallel a sputter-cleaned polycrystalline Au sample with a work function $\Phi$ of $5.3 \mathrm{eV}$ [39]. Work function differences can be determined with high precision $(0.01 \mathrm{eV})$ using this approach. Figure 12(a) shows as an example the energy dependence of the SEY at low energy for a CNT sample and the Au reference, while the derivatives of these curves are shown in Fig. 12(b). As the derivative represents symmetrical peaks, we use their minimum to determine the work function difference. The measured $\Phi$ values are summarized in Table II. After insertion in the analysis system $\Phi$ is around 4.4-4.5 eV. Figure 12(c) indicates that the UHV annealing up to $300{ }^{\circ} \mathrm{C}$ has only a minor influence on the surface work function of the CNTs. As determining absolute work function values is always linked to experimental uncertainties (typically $0.1 \mathrm{eV}$ ), after annealing of the fully covered samples, we have additionally measured

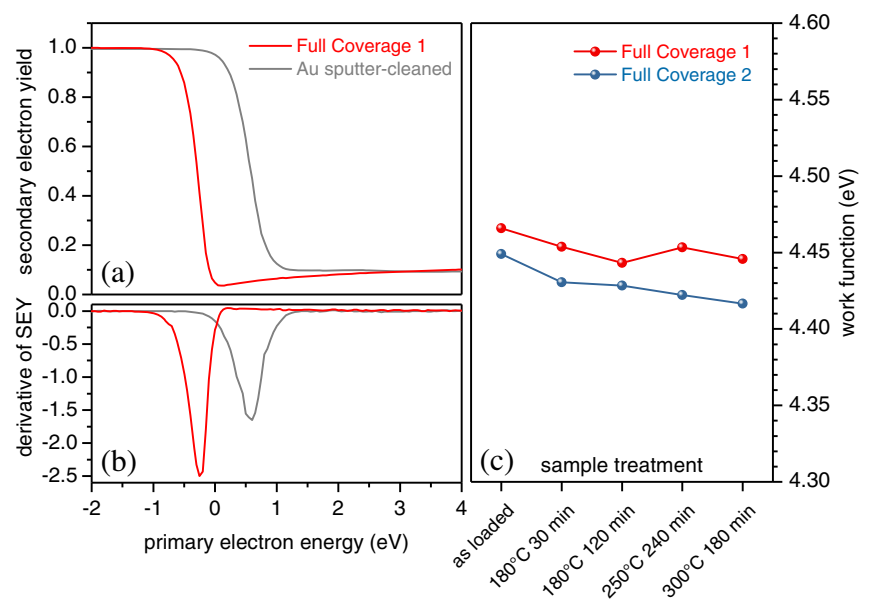

FIG. 12. Work function analysis based on SEY measurements. Example LE-SEY data of the homogeneous CNT sample "Full Coverage 1" and a sputter-cleaned Au reference sample in (a) and the curves derivatives in (b). The variation of the work function during the performed heating cycle of samples "Full Coverage 1" and "Full Coverage 2" under UHV based on SEY measurements is shown in (c). 
TABLE II. Determined work function $\Phi$ in $\mathrm{eV}$ for the four different samples based on SEY and UPS analysis.

\begin{tabular}{lcccc}
\hline \hline Method-sample condition & Honeycomb-like array & Squared-islands array & Full Coverage 1 & Full Coverage 2 \\
\hline SEY-as loaded & $4.38 \pm 0.1$ & $4.40 \pm 0.1$ & $4.47 \pm 0.1$ & $4.45 \pm 0.1$ \\
SEY-after annealing & $\ldots$ & $\ldots$ & $4.45 \pm 0.1$ & $4.42 \pm 0.1$ \\
UPS-after annealing & $\cdots$ & $\ldots$ & $4.54 \pm 0.1$ & $4.57 \pm 0.1$ \\
\hline \hline
\end{tabular}

$\Phi$ by linear extrapolation of the leading edge of secondary electron emission. The resulting values of $(4.54 \pm 0.1) \mathrm{eV}$ and $(4.57 \pm 0.1) \mathrm{eV}$ for sample "Full Coverage 1 " and "Full Coverage 2", respectively, match very well with the numbers obtained by the SEY analysis.

\section{CONCLUSIONS}

In this paper, we have characterized several CNT samples, focusing on two main arrangement geometries, to characterize the properties of CNT arrays and assess whether they can be considered good candidates for being used operationally in ELENA as cold electron field emitters.

The samples tested so far have shown promising results if operated in optimal conditions, i.e., at a pressure $P$ below $1 \times 10^{-8} \mathrm{mbar}$ and after a vacuum bakeout at a temperature $T$ above $200{ }^{\circ} \mathrm{C}$. Additionally, a conditioning process with voltage ramps is strongly advised in order to achieve reproducible results. UHV and bakeout perfectly match the requirements of ELENA, where the nominal pressure is around $10^{-12}$ mbar and bakeout is part of the conditioning procedure.

One of the biggest concerns about CNTs pertain to their lifetime and stability. The performed tests indicate a good stability and the lifetime is promising for the so-called CNT1-type arrays with honeycomb-like geometry. Namely for the array most thoroughly tested we were able to reach more than $1500 \mathrm{~h}$ of emission at different electric fields and without signs of burnout or degradation. The overall current stability matches the requirements of the electron gun of ELENA. Furthermore, with the emission from CNTs only dependent on the electric field, it would also be possible to devise a feedback system adjusting the applied electric field in case of small long-term instabilities. The stability proved so far by the CNT emitters seems to be compatible with such a solution since no significant current drops have been observed if CNTs are operated under optimal conditions.

For the maximum reachable current, all samples show that they can reach the maximum required value of $5 \mathrm{~mA}$. We have successfully reached current densities as high as $2 \mathrm{~mA} / \mathrm{cm}^{2}$ with all samples, which would already suffice for our purpose considering the sample sizes.

The use of a large area cathode would most likely be beneficial for optimizing the performance for operational use, since it would allow us to use a lower electric field. This is beneficial in our particular case since the energy of the required electron beam is extremely low
( 355 and $55 \mathrm{eV}$ ) and therefore the beam would anyway undergo a deceleration process after the emission. However, the tests shown in Figs. 10(a) and 10(b) show how an increase of the applied electric field with consequent increase of the emitted current would not influence the emission stability, while instead permitting lower current fluctuations, at least when considering the time frames $(20 \mathrm{~h})$ and the current densities investigated in this case (up to $1.5 \mathrm{~mA} / \mathrm{cm}^{2}$ ).

The choice of having a large number of CNTs and small channels also seem to improve stability and lifetime. This can explain why the honeycomb-like arrays proved to have the best performance. The choice of this pattern is aimed at having a large number of CNTs emitting simultaneously, and the honeycomb arrangement permits the best space optimization to maximize this parameter. A reason for the poor performance of the square-islands array may derive from this feature, since in this case the total number of CNTs is much smaller compared to the honeycomb-like array of similar chip size. This also means that individual CNTs would need to emit a larger current if the total chip size and current density match the values of a CNT1 array.

The results achieved so far are part of a project aiming at designing an electron gun for the ELENA electron cooler. To achieve this goal, further tests will be planned for the future. Measurements of the beam initial energy are deemed essential to assess whether a CNT cathode can outperform the current thermionic gun used in operation in ELENA. For this purpose, we foresee the commissioning of an additional test bench in triode configuration, consisting of the emitting cathode, a grid and an anode consisting of a phosphor screen to perform such tests. Considering the necessity of having a very homogeneous field in the emission region, while keeping the electric field as low as possible, an extracting grid at appropriate distance, around approximately $1 \mathrm{~mm}$, is considered to be necessary for extracting a low-transverse energy electron beam. Such grid distance, coupled with the electron beam current requirements, imply the application of an electric field of the order of a few $\mathrm{keV}$. Since the electron gun also requires a switching mode to comply with ELENA's beam cycle, the gun must therefore undergo high-voltage switching, which consequently represents a further step toward the final electron gun design.

The use of such gun in the ELENA cooler, aside from hopefully decreasing the electron beam temperature, would also allow simplification of the gun arrangement, eliminating the necessity of a heating filament, thus making the 
conditioning process faster and simpler, while at the same time achieving a more efficient electron cooling process.

\section{ACKNOWLEDGMENTS}

Mechanical parts and electronics realized with the participation of Jean Cenede, Alexandre Frassier, and Wilfried Devauchelle. SY-BI-XEI, CERN. SEM analysis performed with Ana Teresa Perez Fontenla, Alexander Lunt, and Elisa Garcia-Tabares Valdivieso. EN-MME-MM, CERN. This work has been supported by the European Unions Horizon 2020 research and innovation programme under the Marie Skłodowska-Curie Grant agreement No. 721559. J. R. L. acknowledges support by the Generalitat Valencia under Grant agreement No. CIDEGENT/2019/058.

[1] S. Maury, W. Oelert, W. Bartmann, P. Belochitskii, H. Breuker, F. Butin, C. Carli, T. Eriksson, S. Pasinelli, and G. Tranquille, Elena: The extra low energy anti-proton facility at CERN, Hyperfine Interact. 229, 105 (2014).

[2] C. Alanzeau et al., Extra low energy antiproton ring (ELENA) and its transfer lines: Design Report, CERN2014-002, 2014.

[3] W. Bartmann, P. Belochitskii, H. Breuker, F. Butin, C. Carli, T. Eriksson, W. Oelert, R. Ostojic, S. Pasinelli, and G. Tranquille, The ELENA facility, Phil. Trans. R. Soc. A 376, 20170266 (2018).

[4] S. Maury, Design study of the antiproton decelerator: Ad, CERN/PS 96-43 (AR), 1996.

[5] G. I. Budker, An effective method of damping particle oscillations in proton and antiproton storage rings, Sov. At. Energy 22, 438 (1967).

[6] I. N. Meshkov, Electron cooling: Status and perspectives, CERN Repository, 1994.

[7] H. Poth, Electron cooling: Theory, experiment, application, CERN Repository, 1990.

[8] V. V. Parkhomchuk and A. N. Skrinskii, Electron cooling: 35 years of development, Phys. Usp. 43, 433 (2000).

[9] G. Tranquille, A. Frassier, and L. Joergensen, The ELENA electron cooler: Parameter choice and expected performance, in Proceeding of COOL2013, Murren, Switzerland (2014), pp. 133-135, WEPPO16, ISBN 978-3-95450-140-3.

[10] G. Tranquille and J. Cenede, Design and optimization of the ELENA electron cooler gun and collector, in Proceeding of IPAC2016 (2016), THPMB048, https://dx.doi.org/ 10.18429/JACoW-IPAC2016-THPMB048.

[11] R.H. Fowler and L. Nordheim, Electron emission in intense electric fields, Proc. R. Soc. A 119, 173 (1928).

[12] Y. Saito, Carbon Nanotube and Related Field Emitters (Wiley-VCH, New York, 2010).

[13] Y. Saito, Carbon Nanotube and Related Field Emitters: Fundamentals and Applications (Wiley-VCH, New York, 2010).

[14] C. Li, Y. Zhang, M.Mann, D. Hasko, W. Lei, B. Wang, D. Chu, D. Pribat, G. Amaratunga, and W. I. Milne, High emission current density, vertically aligned carbon nanotube mesh, field emitter array, Appl. Phys. Lett. 97, 113107 (2010).

[15] Y. M. Wong, W. P. Kang, J. L. Davidson, B. K. Choi, W. Hofmeister, and J. H. Huang, Array geometry, size and spacing effects on field emission characteristics of aligned carbon nanotubes, Diam. Relat. Mater. 14, 2078 (2005).

[16] S. H. Jo, Y. Tu, Z. P. Huang, D. L. Carnahan, D. Z. Wang, and Z.F. Ren, Effect of length and spacing of vertically aligned carbon nanotubes on field emission properties, Appl. Phys. Lett. 82, 3520 (2003).

[17] R. C. Smith and S. R. P. Silva, Maximizing the electron field emission of carbon nanotube arrays, Appl. Phys. Lett. 94, 133104 (2009).

[18] N. Hong, K. H. Koh, S. Lee, P. N. Minh, N. T. T. Tam, and P. H. Khoi, Comparison of field electron emission from different carbon nanotube array structures, J. Vac. Sci. Technol. B 27, 749 (2009).

[19] Y. Cheng and O. Zhou, Elecron field emission from carbon nanotubes, C.R. Phys. 4, 1021 (2003).

[20] J. T. L. Thong, C. H. Oon, W. K. Eng, W. D. Zhang, and L. M. Gan, High-current field emission from a vertically aligned carbon nanotube field emitter array, Appl. Phys. Lett. 79, 2811 (2001).

[21] J. I. Sohn, S. Lee, Y.-H. Song, S.-Y. Choi, K.-I. Cho, and K.-S. Nam, Patterned selective growth of carbon nanotubes and large field emission from vertically well-aligned carbon nanotube field emitter arrays, Appl. Phys. Lett. 78, 901 (2000).

[22] https://www.ni.com/pdf/manuals/320999e.pdf.

[23] https://www.nano-lab.com.

[24] L. Sun, G. Yuan, L. Gao, J. Yang, M. Chhowalla, M. H. Gharahcheshmeh, K. K. Gleason, Y. S. Choi, B. H. Hong, and Z. Liu, Chemical vapour deposition, Nat. Rev. 1, 5 (2021).

[25] V. Petit, M. Taborelli, H. Neupert, P. Chiggiato, and M. Belhaj, Role of the different chemical components in the conditioning process of air exposed copper surfaces, Phys. Rev. Accel. Beams 22, 083101 (2019).

[26] H. Todokoro, N. Saitou, and S. Yamamoto, Role of ion bombardment in field emission current instability, Jpn. J. Appl. Phys. 21, 1513 (1982).

[27] C. M. Collins, R. J. Parmee, W. I. Milne, and M. T. Cole, High performance field emitters, Adv. Sci. 3, 1500318 (2016).

[28] R. G. Forbes, Comments on the continuing widespread and unnecessary use of a defective emission equation in field emission related literature, J. Appl. Phys. 126, 210901 (2019).

[29] P. Yaghoobi, K. Walus, and A. Nojeh, First-principles study of quantum tunneling from nanostructures: Current in a single-walled carbon nanotube electron source, Phys. Rev. B 80, 115422 (2009).

[30] S. Han and J. Ihm, First-principles study of field emission of carbon nanotubes, Phys. Rev. B 66, 241402(R) (2002).

[31] B. Everitt, The Cambridge Dictionary of Statistics (Cambridge University Press, Cambridge, England, 1998).

[32] C. Kim, Y. S. Choi, S. M. Lee, J. T. Park, B. Kim, and Y. H. Lee, The effect of gas adsorption on the field emission 
mechanism of carbon nanotubes, J. Am. Chem. Soc. 124, 9906 (2002).

[33] A. Kumar, S. Parveen, S. Husain, M. Zulfequar, Harsh, and M. Husain, Oxygen and nitrogen doping in single wall carbon nanotubes: an efficient stable field emitter, J. Alloys Compd. 711, 85 (2017).

[34] H. Ago, W. R. S. T. Kugler, F. Cacialli, M. S. P. Shaffer, A. H. Windle, and R.H. Friend, Work functions and surface functional groups of multiwall carbon nanotubes, J. Phys. Chem. B 103, 8116 (1999).

[35] P. Liu, Q. Sun, F. Zhu, K. Liu, K. Jiang, L. Liu, Q. Li, and S. Fan, Measuring the work function of carbon nanotubes with thermionic method, Nano Lett. 8, 647 (2008).
[36] M. Shiraishi and M. Ata, Work function of carbon nanotubes, Carbon 39, 1913 (2001).

[37] G. Rovida, F. Pratesi, M. Maglietta, and E. Ferroni, Chemisorption of oxygen on the silver (111) surface, Surf. Sci. 43, 230 (1974).

[38] R. Cimino, L. A. Gonzalez, R. Larciprete, A. Di Gaspare, G. Iadarola, and G. Rumolo, Detailed investigation of the low energy secondary electron yield of technical cu and its relevance for the LHC, Phys. Rev. ST Accel. Beams 18, 051002 (2015).

[39] H. Kawano, Effective work functions for ionic and electronic emissions from mono- and polycrystalline surfaces, Prog. Surf. Sci. 83, 1 (2008). 